http://jmscr.igmpublication.org/home/ ISSN (e)-2347-176x ISSN (p) 2455-0450 crossref DOI: https://dx.doi.org/10.18535/jmscr/v8i1.16

\title{
Serum Ferritin: A Biomarker to predict Disease Severity in Patients with Dengue Infection
}

\author{
Authors \\ Dr S A Kanitkar, Dr Murukoti Sonika Reddy, Dr Anu Gaikwad, \\ Dr Vaja Mukhtarahmed, Dr Sura Sreekar, Dr Priyanka Reddy Produturu, \\ Dr Mankoskar Yash Surendrakumar \\ Department of General Medicine Dr D. Y. Patil Medical College, Hospital and Research Centre, \\ PIMPRI, Pune, India \\ *Corresponding Author \\ Dr Murukoti Sonika Reddy
}

\begin{abstract}
Background: Each year, up to 400 million people are infected and 22,000 die from severe dengue globally. An important cause of acute febrile illness especially in tropics and subtropics in the world is dengue fever. It is approximated that one million dengue cases are reported to WHO from these regions. Aim of the study was early detection of dengue severity using ferritin as a biomarker in dengue patients.

Methods: A retrospective study was conducted on 50 patients in Department of Medicine from November 2018 to December 2019. Data of all patients admitted with dengue were collected and analysed with special reference to serum ferritin.

Results: In 50 patients, majority were in the age group of 20-40 years. Mean ferritin level was much higher in severe dengue patients i.e. $861.28 \pm 183.17$ as compared to patients with non severe dengue i.e. $400 \pm 151.7$. Serum ferritin levels was high in patients with severe dengue as compared to non severe forms.

Conclusion: Serum ferritin levels may serve as a biomarker to predict dengue disease severity

Keywords: severe dengue, serum ferritin, dengue fever.
\end{abstract}

\section{Introduction}

Dengue, a mosquito borne viral infection has become a significant public health problem with an estimated 3.9 billion people in 128 countries being at risk of infection. ${ }^{[1,2]}$ Each year, up to 400 million people are infected and 22,000 die from severe dengue globally. ${ }^{[3]}$ An important cause of acute febrile illness especially in tropics and subtropics in the world is dengue fever. It is approximated that one million dengue cases are reported to WHO from these regions. ${ }^{[4]}$ The dengue virus which has four serotypes is transmitted to humans by Aedes mosquitoes especially Aedes aegypti. The four serotypes of dengue virus cause anywhere from asymptomatic infection to severe dengue. ${ }^{[5]}$

The illness is divided into 3 distinct phases $\{1\} \mathrm{A}$ nearly febrile phase was lasting from day 1 to day $7\{2\}$ A critical phase from day 3 to day $8\{3\}$ Convalescent-phase.

The dengue illness can proceed to potential complications like dengue haemorrhagic fever 
(DHF) and dengue shock syndrome (DSS), both defined as severe dengue by WHO. The commonly used investigations to identify the illness are NS1 ANTIGEN (non-structural protein 1), IgM and IgG by MAC-ELISA/RAPID KIT TEST. ${ }^{[6,7]}$ Clinically dengue fever is suspected when acute febrile illness of 2-7 days presents with two or more than two of the following, namely, headache, retroorbital pain, myalgia, arthralgia, rash, and haemorrhagic manifestations ${ }^{[8]}$ Ferritin is an acute phase reactant released by the reticuloendothelial cells. Hyperferritinemia is associated with immune activation and coagulation disturbances. ${ }^{[9]}$ Currently, no tests are available to monitor or predict severity and outcome of dengue, present study was aimed to validate serum ferritin level as a marker of severe dengue as compared to nonsevere dengue.

\section{Methodology}

This was a retrospective study conducted on 50 patients in Department of Medicine, Dr. D.Y.Patil Medical and Research Centre, Pune from November 2018 to December 2019. The data of all patients admitted with dengue at Dr D.Y.Patil Medical and Research Centre, Pune were collected and analysed. Patients admitted were evaluated for CBC, Ferritin, TROP T, CPKMB, SGOT, SGPT, Albumin levels, Chest X-Ray, USG, ECG and 2D Echo.

\section{Inclusion Criteria}

Patients admitted with the diagnosis of Dengue fever on the basis of history, clinical examination and positive non-structural protein 1 (NS1) antigen or positive $\operatorname{IgM}$ antibodies for Dengue were included in this study.

\section{Exclusion Criteria}

Patients having microcytic anaemia, presenting with DHF or DSS were excluded, Patients other than dengue presenting as acute febrile illness were excluded and pregnant women were also excluded.

\section{Statistical Analysis}

The data was entered in Microsoft Excel 2010 version spreadsheet and analysis was done by EPI INFO (version 7) provided by CDC Atlanta. Tables were prepared for the variables. Pearson chi-square test was used for categorical variables in the study. Results on continuous measurements are presented on mean \pm SD. In our study ' $p$ ' value less than 0.05 was considered to be significant.

The ethical clearance was obtained from the Institutional Ethical committee.

\section{Procedure}

Patients were divided into two groups on the basis of ferritin levels.

Normal levels of serum ferritin were considered between 24-300ng/dl.

Patients having levels more than 600ng/dl were considered high serum ferritin levels.

Group A consisted of patients with ferritin level $<600 \mathrm{ng} / \mathrm{dl}$ and those $>600 \mathrm{ng} / \mathrm{dl}$ were included in Group B. Severe dengue was defined as patients having warning signs like mucosal bleeding, increasing haematocrit, decreasing platelet count, fluid accumulation, abdominal pain, severe plasma leakage, severe bleeding and organ failure.

Discharge from the hospital without complications or developing severe dengue during the hospital stay was taken as end point of study

Dengue IgG,IgM and NS1 was estimated by BENESPHERA ELISA Microplate Washer W21,BENESPHERA ELISA Microplate Reader and E21 LISAWASH 4000 ELISA Microplate Washer.

Serum ferritin level of all the patients was sent to pathology department and was measured by ELISA on the day of admission.

\section{Results}

This study was conducted on 50 patients admitted with diagnosis of dengue fever

Following Graph and Tables show the observations of the study 
Graph 1: Gender distribution

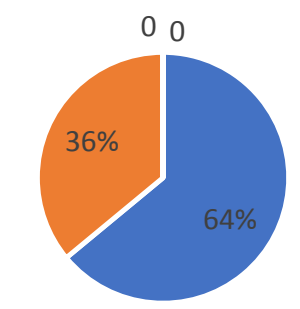

- Males a Females

Out of the total 50 patients, $64 \%$ were males i.e. 32 patients and $36 \%$ were femalesi.e. 18 patients.

Table 1: Age wise distribution

\begin{tabular}{|l|c|c|c|}
\hline Age group & $<20 y r s$ & $20-40 y r s$ & $>40 y r s$ \\
\hline Male & 8 & 20 & 4 \\
\hline Female & 1 & 13 & 4 \\
\hline
\end{tabular}

Of 50 patients, majority were in the age group of 20-40 years followed by less than 20 years and more than 40 years respectively.

$66 \%$ (33) patients were in 20- 40 years, $18 \%$ (9) patients in $<20$ years and $16 \%$ (8) patients in $>40$ years.

In this study, Mean patient age was $31.5 \pm 11.2$ years.

Mean duration of hospital stay was $4 \pm 1.12$ days.

Mean duration of fever was $5.3 \pm 1.81$ days.

Table 2: Gender wise distribution of serum ferritin levels

\begin{tabular}{|l|c|c|c|}
\hline Serum ferritin(ng/dl) & male & female & Total \\
\hline$<600$ & 17 & 13 & 30 \\
\hline$>600$ & 15 & 5 & 20 \\
\hline Total & 32 & 18 & 50 \\
\hline
\end{tabular}

In Patients with serum ferritin levels <600ng/dl, 17 were males and 13 were females and serum ferritin levels >600ng/dl, 15 were males and 5 were females.

Table 3: Patient wise distribution of serum ferritin levels

\begin{tabular}{|l|c|}
\hline Serum ferritin levels & Number of patients \\
\hline$<300$ & 2 \\
\hline $300-400$ & 16 \\
\hline $401-500$ & 7 \\
\hline $501-600$ & 5 \\
\hline $601-700$ & 4 \\
\hline $701-800$ & 8 \\
\hline $801-1000$ & 6 \\
\hline$>1000$ & 2 \\
\hline
\end{tabular}

This table shows serum ferritin levels ranging from $<300$ to $>1000 \mathrm{ng} / \mathrm{dl}$ in the study subjects.

Mean ferritin level was much higher in severe dengue patients i.e. $861.28 \pm 183.17$ as compared to patients with non severe dengue fever i.e. $400 \pm$ 151.7.

Table 4: Association between serum ferritin level and severe dengue illness

\begin{tabular}{|l|c|c|c|}
\hline $\begin{array}{l}\text { Severe } \\
\text { dengue }\end{array}$ & $\begin{array}{c}\text { Serum ferritin } \\
(<600 \mathrm{ng} / \mathrm{dl})\end{array}$ & $\begin{array}{c}\text { Serum ferritin } \\
(>600 \mathrm{ng} / \mathrm{dl})\end{array}$ & P value \\
\hline No & 28 & 4 & \\
\hline Yes & 2 & 16 & 0.001 \\
\hline
\end{tabular}

Chi square test was used to compare categorical variables and to predict the correlation between serum ferritin and dengue severity.

This table signifies that serum ferritin levels was high in patients with severe dengue at the time of admission compared to non severe forms which was statistically significant with a $\mathrm{p}$ value of $<0.05$.

In group A of 30 patients, 2 had severe dengue with serum ferritin levels of $<600 \mathrm{ng} / \mathrm{dl}$; whereas in group B,out of 20 patients 16 had severe dengue with serum ferritin levels of $>600 \mathrm{ng} / \mathrm{dl}$.

Out of total 18 severe dengue patients, all of them had plasma leakage in the form of ascites, pleural effusion and pseudo gall bladder wall thickening. Platelet count levels dropped to 20,000 in 13 patients, remaining 5 patients platelet count dropped till 30,000.

2 patients had dengue myocarditis. 15 patients had dengue hepatitis.3 patients had mucosal bleeding and no one had DSS.

\section{Discussion}

In a study conducted by Selvamuthukumaran S, the majority of patients belonged to the male gender $59.19 \%$. Mean hospital stay duration was $5 \pm 2$ days. 12 patients out of 30 with ferritin levels 200-300ng/dl developed severe dengue, whereas 30 patients out of 49 patients developed severe dengue with ferritin levels 300-400ng/dl and 46 out of 62 patients developed severe dengue with ferritin levels $400-500 \mathrm{ng} / \mathrm{dl}$ and 6 patients who 
had ferritin levels 500-600ng/dl had severe dengue. ${ }^{[4]}$

In my study, out of the total 50 patients, $64 \%$ were males (majority) and 36\% were females. Mean duration of hospital stay was $4 \pm 1.12$ days and out of 30 patients, 2 developed severe dengue with serum ferritin levels of $<600 \mathrm{ng} / \mathrm{dl}$ and 16 out of 20 patients developed severe dengue with serum ferritin levels of $>600 \mathrm{ng} / \mathrm{dl}$.

In a study by Nadeem $M$ et al. Mean patient age was $30.7 \pm 13.8$ years. Mean duration of fever was $5.6 \pm 1.3$ days. Mean duration of hospital stay was $3.7 \pm 1.02$ days. In $70 \%$ patient's serum ferritin level was found $>100 \mu \mathrm{g} / \mathrm{dl}$, whereas only $30 \%$ had ferritin level $\leq 100 \mu \mathrm{g} / \mathrm{dl}$. Mean ferritin levels were much higher in patients with severe dengue i.e. $317.54 \pm 109.52$ as compared to the patients with uncomplicated dengue fever i.e. $168.69 \pm$ 130.7. ${ }^{[10]}$

In my study, mean patient age was $31.5 \pm 11.2$ years. Mean duration of fever was $5.3 \pm 1.81$ days. Mean ferritin level was much higher in severe dengue patients i.e. $861.28 \pm 183.17$ as compared to patients with non severe dengue i.e. $400 \pm$ $151.7 .60 \%$ patients had serum ferritin values more than $600 \mathrm{ng} / \mathrm{dl}$ and $40 \%$ patients had less than $600 \mathrm{ng} / \mathrm{dl}$.

In a study by Ahmed $\mathrm{A}$ et al. showed mean duration of fever at presentation was $5.49 \pm 2.65$ days and mean duration of hospital stay was 2.73 \pm 1.41 days. $^{[11]}$

Study conducted by Soundravally $\mathrm{R}$ et al. concluded that raised ferritin levels could predict the dengue severity with sensitivity of $76.9 \%$ and specificity of $83.3 \%$ on the day of admission. ${ }^{[12]}$

\section{Conclusion}

Raised serum ferritin levels are significantly associated with severe dengue. Mean ferritin levels are also high in patients with severe dengue as compared to dengue fever.

Serum ferritin levels on the day of admission may serve as biomarker for an early prediction of disease severity in dengue virus infection.

\section{Limitation}

A major limitation of this study was small sample size.

\section{Abbreviations}

WHO- World Health Organization

DHF- Dengue Haemorrhagic Fever

DSS- Dengue Shock Syndrome

CBC- Complete Blood Count

USG- Ultra Sonography

ECG- Electro Cardio Gram

SGOT- Serum Glutamic Oxalo Acetic

Transaminase

SGPT- Serum Glutamic Pyruvic Transaminase

SD- Standard Deviation

\section{References}

1. Yacoub, S. and B. Wills (2014). Predicting outcome from dengue. BMC Med. 2014;12(1):147.

2. Brady O, Gething $\mathrm{P}$, Bhatt $\mathrm{S}$, Messina $\mathrm{J}$, Brownstein J, Hoen A, et al. Refining the global spatial limits of dengue virus transmission by evidence-based consensus. PLoSNegl Trop Dis. 2012;6(8):e1760.

3. Centers for Disease Control and Prevention. About Dengue: What You Need to Know. https://www.cdc.gov/dengue/about/index.h tml. Accessed 12 May 2019.

4. Selvamuthukumaran S. Severity of Dengue Fever and Serum Ferritin LevelsA Correlative Study in a Rural Tertiary Care Medical College and Hospital in Tamil Nadu (South India). Ann. Int. Med. Den. Res. 2018; 4(3):ME52-ME54.

5. Back AT and Lundkvist A, Dengue viruses - an overview. Infect Ecol Epidemiol 2013; 3; 10 -13. 4.

6. Wang SM. Sekaran SD, Early diagnosis of Dengue infection using a commercial dengue Duo rapid test kit for the detection of NSI, IgM, IgG, AM J troMedHyg 2010; 83: 690-95. 5. 
7. Samanta J and Sharma V, Dengue and its effects on liver. World J Clin cases 2015; $3 ; 125-31$

8. National Guidelines for Clinical Management of Dengue Fever, National Vector Bourne Disease Control Programme, 2015, http://www.nvbdcp.gov.in/Doc/Clinical\%2 0Guidelines.pdf.

9. Soumyabrata Roy chauduri et al. Serum ferritin a backstage weapon in Diagnosis of Dengue fever: Interdisciplinary perspectives on infectious diseases, Vol (2017), article Id : 7463489:

10. Nadeem M, Shafiq MM, Manzoor MS, Ahmed SI. Serum Ferritin: An Indicator of Disease Severity in Patients with Dengue Infection. Journal of Rawalpindi Medical College (JRMC). 2016 Sep 30;20(3):1657.

11. Ahmed A, Alvi AH, Butt A, Nawaz AA, Hanif A, Assessment of Dengue fever severity through liver function tests. J Coll Physicians Surg Pak 2014; 24: 640-44

12. Soundravally R, Agieshkumar B, Daisy M, Sherin J, Cleetus CC. Ferritin levels predict severe dengue. Infection 2015 ;43:13-19. 